\title{
Percutaneous puncture and embolisation for pancreatitis-related pseudoaneurysm: the feasibility of thrombin injection even in collection of fluid surrounding the pseudoaneurysm
}

\author{
Kenkichi Michimoto ${ }^{1 \mathrm{AB}, \mathrm{BD}, \mathrm{E}, \mathrm{F}}$, Takahiro Higuchi ${ }^{1 B, D}$, Keitaro Enoki ${ }^{1 B, F}, Y_{0}$ Matsui $^{10}$, Shinsuke Takenaga ${ }^{1 D}$, \\ Chisato Saeki ${ }^{2 B, D}$ \\ 'Department of Radiology, Fuji City General Hospital, Shizuoka, Japan \\ ${ }^{2}$ Department of Internal Medicine, Fuji City General Hospital, Shizuoka, Japan
}

\section{Abstract}

Purpose: Pancreatitis-related pseudoaneurysm, a potentially life-threatening condition, is treated utilising endovascular management as a first choice and alternatively by percutaneous direct puncture of the aneurysm and embolisation.

Case report: A 50-year-old man with alcohol-induced necrotic pancreatitis underwent transcatheter arterial embolisation (TAE) for multiple pancreatic pseudoaneurysms. TAE failed in one enlarged aneurysm in the pancreatic body, and percutaneous direct needle puncture and coagulation using thrombin was planned. Precise puncture of the aneurysmal sac under fluoroscopy and computed tomographic (CT) guidance failed, but we could inject about 500 units of thrombin through the outer cannula into the collection of fluid surrounding the aneurysm. Compared to preoperative images, contrast-enhanced CT (CECT) showed shrinkage of the pseudoaneurysm without complications just after the procedure and its disappearance five days after the procedure.

Conclusions: Percutaneous direct puncture and embolisation is a feasible choice to treat pancreatic pseudoaneurysms, and injection of thrombin even into the collection of fluid surrounding the pseudoaneurysm can be a viable alternative, especially in cases in which precise puncture of the aneurysmal sac is difficult because of its size and location.

Key words: aneurysm, pancreatitis, thrombin.

\section{Introduction}

Pancreatic pseudoaneurysm related to pancreatitis is well understood and potentially fatal. Proteolytic activity of infused pancreatic fluid and local inflammation compromise adjacent vessel walls, which leads to the development of pseudoaneurysm in peri-pancreatic arteries [1]. Prompt treatment following radiological diagnosis regardless of the size of the pseudoaneurysm or symptoms of the patient avoids potential spontaneous rupture and catastrophic haemorrhage [1].

Endovascular management, such as transcatheter arterial embolisation (TAE), is commonly the first choice to treat pancreatic pseudoaneurysms [1], but percutaneous direct puncture for pancreatitis-related pseudoaneurysm and coagulation using thrombin [1-3], coils [1,4], or glue $[1,4]$ has also been reported, often performed after a failed attempt at TAE.

The small size of our patient's pseudoaneurysm and its location in the pancreatic body made endovascular management and precise percutaneous puncture of the aneurysmal sac difficult, but we could perform percutaneous puncture and inject thrombin into the collection of fluid surrounding the pseudoaneurysm without complication. We believe this is the first case report demonstrating the feasibility of thrombin injection into the fluid

Correspondence address:

Dr. Kenkichi Michimoto, Department of Radiology, Fuji City Genaral Hospital, 50 Takashimachou, 417-8567, Shizuoka, Japan, e-mail: kenkichi.m@gmail.com

Authors' contribution:

A Study design · B Data collection · C Statistical analysis · D Data interpretation · E Manuscript preparation · F Literature search · G Funds collection 

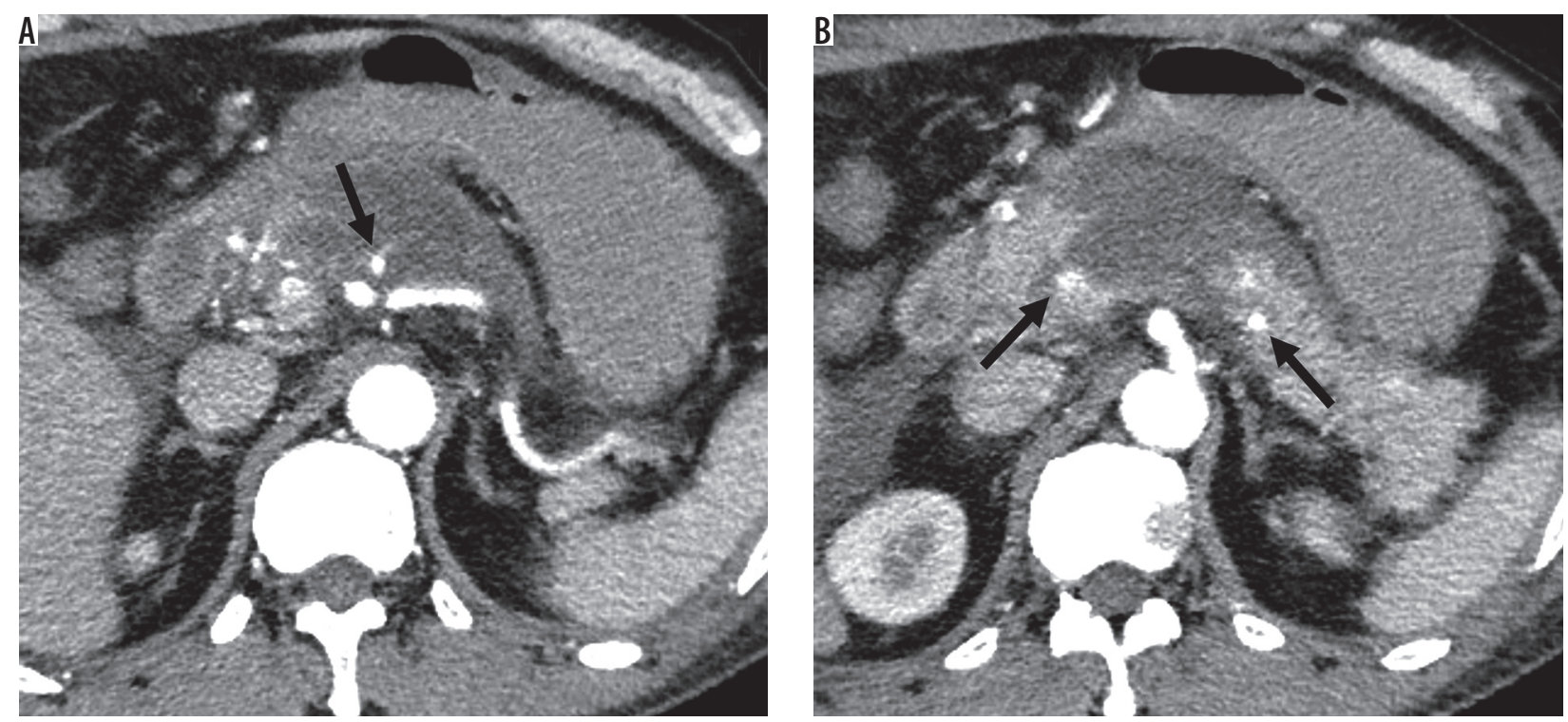

Figure 1. Contrast-enhanced computed tomography obtained one week after the patient's hospital admission demonstrated pancreatitis with acute necrotic collection and multiple pseudoaneurysms in the pancreatic head, body, and tail (arrow)

surrounding the pseudoaneurysm and not the aneurysmal sac.

\section{Clinical presentation}

A 50-year-old man admitted to our hospital for alcoholinduced acute pancreatitis with elevated serum amylase (1756 U/l) underwent contrast-enhanced computed tomography (CECT) one week after admission, which demonstrated necrosis of the pancreatic body with acute necrotic collection (ANC) and multiple pseudoaneurysms in the pancreatic head, body, and tail (Figure 1). The patient remained haemodynamically stable throughout his

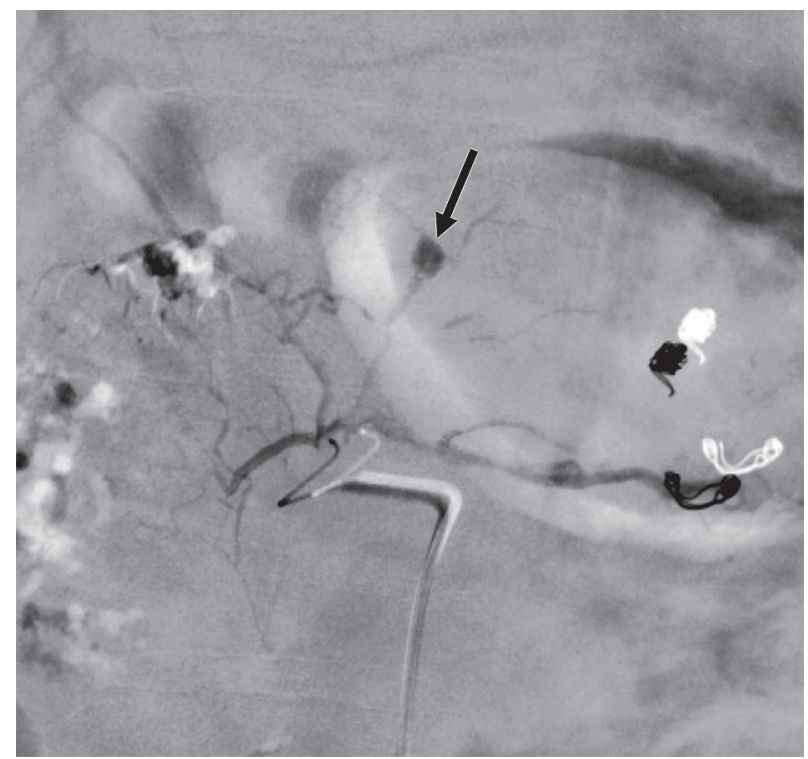

Figure 2. Selective embolisation for the aneurysm in the pancreatic body (arrow) fed by the dorsal pancreatic artery arising from the proximal portion of the superior mesenteric artery failed course, but his haemoglobin level decreased from 16.7 to $12.5 \mathrm{~g} / \mathrm{dl}$. Initial TAE of the pseudoaneurysms in the pancreatic head supplied from the gastroduodenal artery and the pancreatic tail supplied from the splenic artery utilising coils and n-Butyl cyanoacrylate was successful, but one remaining aneurysm in the pancreatic body fed by the dorsal pancreatic artery arising from the proximal portion of the superior mesenteric artery could not be embolised because selective cannulation into the dorsal pancreatic artery had failed (Figure 2).

Follow-up CECT four days after the first TAE demonstrated enlargement of the pseudoaneurysm from 4 to $10 \mathrm{~mm}$ in diameter, and a second attempt at TAE failed. We therefore planned percutaneous direct needle puncture with a 21-gauge coaxial needle of the pseudoaneurysm, which was surrounded by ANC, under fluoroscopy or CT guidance because of poor visualisation offered under ultrasonography guidance. We were unsuccessful in our initial attempt to puncture the pseudoaneurysm under local anaesthesia using fluoroscopy guidance with a 4-Fr shepherd hook-shaped catheter and microcatheter placed in the celiac artery as a guidepost, so we obtained percutaneous direct imaging of the ANC and main pancreatic duct instead (Figure 3) and attempted to puncture the pseudoaneurysm under CT guidance in the same way. Although placement of the puncture needle adjacent to the pseudoaneurysm prevented any appearance at its tip of arterial backflow from the outer cannula, we confirmed distribution of the injected diluted contrast media into the ANC surrounding the aneurysm rather than into the parent arteries, such as the celiac artery, and then injected about 500 units of thrombin (Fuji Pharma Co., Ltd., Tokyo, Japan) through the outer cannula (Figure 4). Immediately after the thrombin injection, the patient complained of transient abdominal pain that resolved within seconds without treatment. 
Relative to the appearance of the pseudoaneurysm in the pancreatic body on preoperative imaging, CECT obtained just after the procedure showed its shrinkage without complication, such as thrombosis of the parent artery, and it had completely disappeared on CECT obtained five days after the procedure (Figure 5). The patient remained stable after the procedure and was transferred to another hospital for chronic-stage treatment.

\section{Discussion}

Percutaneous direct injection of thrombin, although an off-label use of the thrombin, has proved a effective and minimally invasive procedure primarily in the treatment of iatrogenic superficial pseudoaneurysms, such as those in the femoral and brachial arteries $[5,6]$. Commonly, the tip of a fine needle, such as a 21- or 22-gauge spinal needle, is inserted into the centre of the aneurysmal sac under B-mode ultrasonography guidance, and a small amount of thrombin is slowly injected into the sac under colour-flow
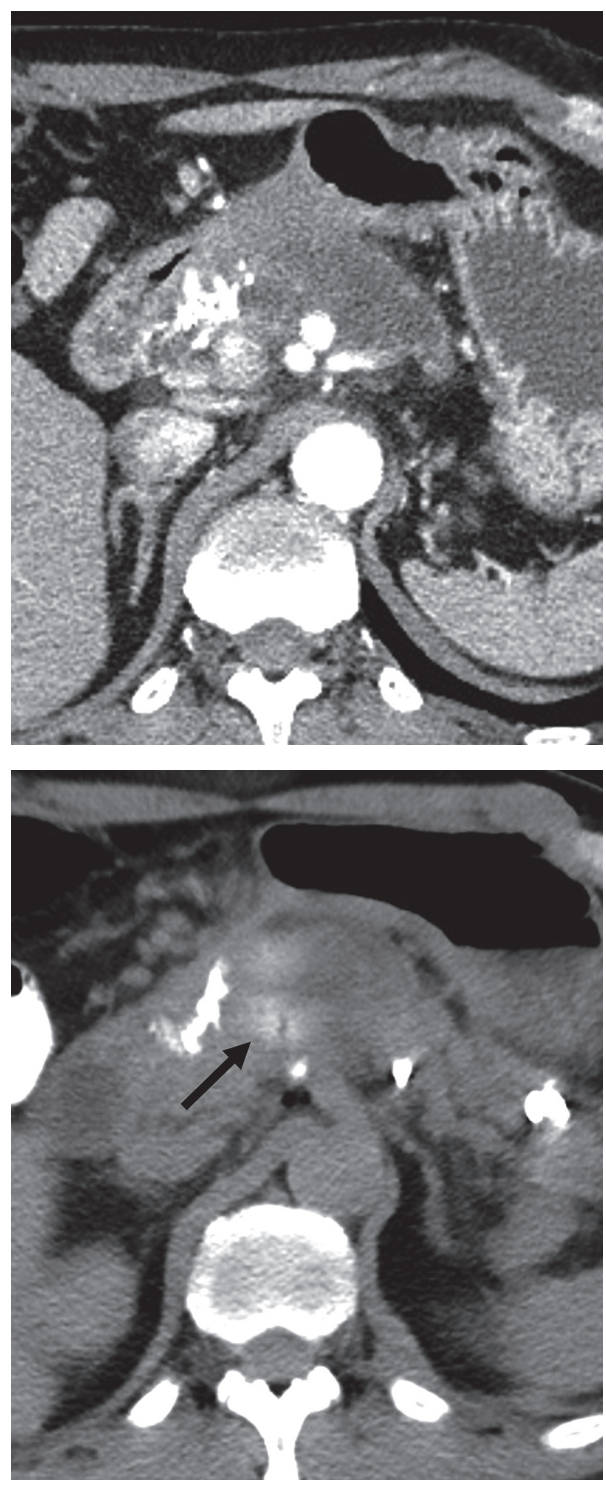

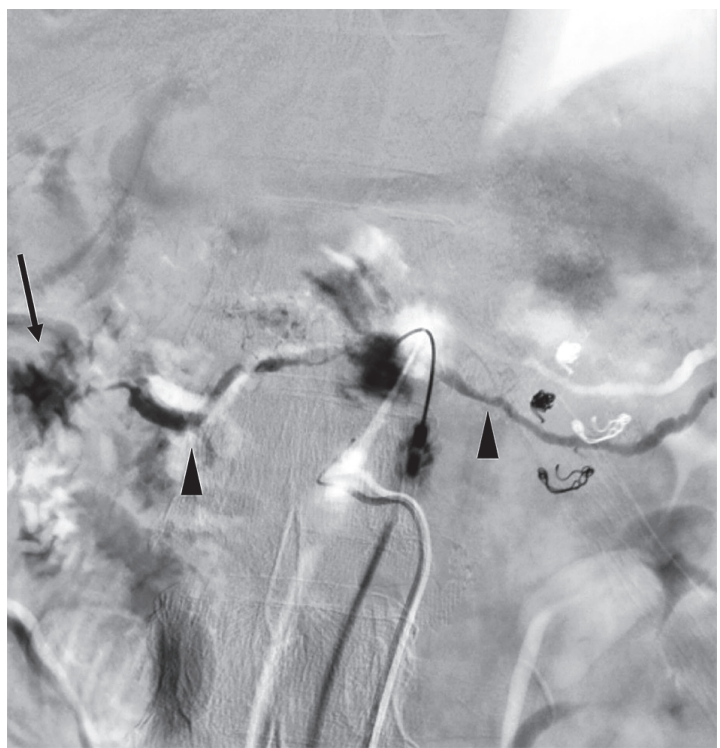

Figure 3. Direct puncture by a 21-gauge needle under fluoroscopy guidance demonstrated perfusion of the fluid collection surrounding the aneurysm, main pancreatic duct (arrowhead), and duodenum (arrow)

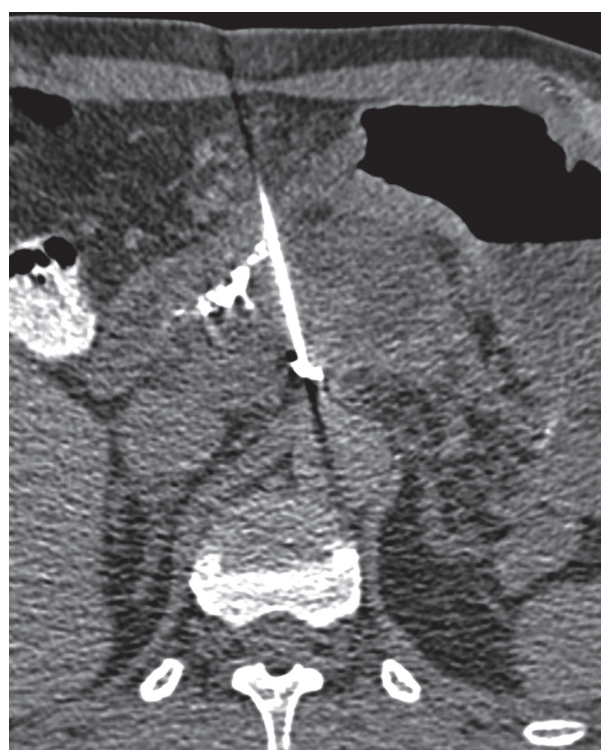

Figure 4. A) Direct puncture under computed tomography guidance for the enlarged pseudoaneurysm. B) The tip of the puncture needle was located immediately adjacent to the aneurysm. C) The injected contrast media and thrombin distributed in fluid collection around the aneurysm (arrow) 

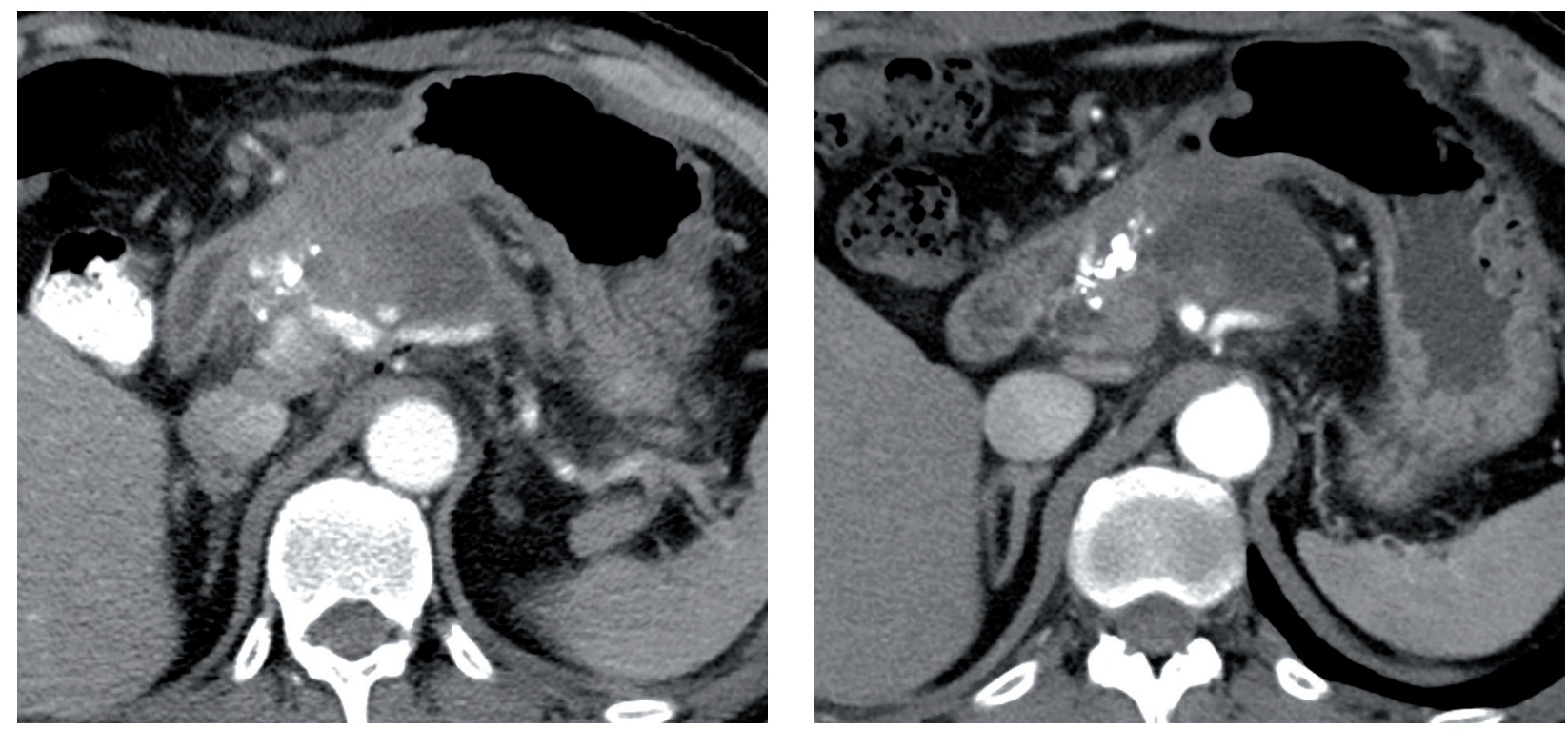

Figure 5. Contrast-enhanced computed tomography demonstrated shrinkage of the aneurysm just after the procedure (A) and its disappearance after 5 days (B)

ultrasonography monitoring [4-6]. Other imaging guidance, such as fluoroscopy [4] or CT [6], can be employed in cases with visceral pseudoaneurysms according to the quality of visualisation of the aneurysm. The injected thrombin converts inactive fibrinogen into fibrin to form a thrombus in the pseudoaneurysm. The likelihood of thrombosis is not affected by the size of the aneurysmal dome or neck or anticoagulation status of the patient [6]. Potential risks of this procedure include thrombosis of the parent artery, peripheral thromboembolism, and allergic reactions $[1,5,6]$.

We believe this is the first report that suggests the feasibility of injection of thrombin into the fluid collection around the pseudoaneurysm and not the aneurysmal sac itself. This injected thrombin may infiltrate the pseudoaneurysm through a false vascular wall comprising fibrous and connective tissue rather than normal arterial tissue and then coagulate the arterial flow in the pseudoaneurysm. Certainly, injection of thrombin directly into the aneurysmal sac rather than into the fluid surrounding the pseudoaneurysm leads to a more reliable coagulation effect, but our procedure offers the next best way to coagulate in cases in which precise puncture of the aneurysm is difficult because of the small size, deep location, or poor visualisation with ultrasonography or CT fluoroscopy of the pseudoaneurysm. Moreover, compared with the direct injection of thrombin into the aneurysmal sac, our procedure reduces the risk of complications such as coagulation in the parent artery and peripheral thromboembolism.

Although pseudoaneurysms bear the risk of spontaneous thrombosis, such thrombosis cannot be predicted [5], and we believe the pseudoaneurysm of our case was unlikely to resolve spontaneously because it continued to grow in size until the procedure.

\section{Conclusions}

Injection of thrombin even into the collection of fluid surrounding a pancreatic pseudoaneurysm can serve as a feasible alternative treatment to percutaneous puncture and embolisation, especially in cases in which precise puncture of the aneurysmal sac is difficult because of the size or location of the pseudoaneurysm.

\section{Conflict of interest}

The authors report no conflicts of interest.

\section{References}

1. Zabicki B, Limphaibool N, Holstad MJV, et al. Endovascular management of pancreatitis-related pseudoaneurysms: a review of techniques. PLoS One 2018; 13: e0191998.

2. Spezia L, Sozzi C, Contro A, et al. Successful post-pancreatitis pseudoaneurysm coagulation by percutaneous computed tomography (CT)-guided thrombin injection. Pol J Radiol 2017; 82: 24-27.

3. McErlean A, Looby S, Lee MJ. Percutaneous ultrasound-guided thrombin injection as first-line treatment of pancreatic pseudoaneurysm. Cardiovasc Intervent Radiol 2007; 30: 526-528.
4. Shrivastava A, Rampal JS, Reddy DN, et al. Direct needle puncture and embolization of splenic artery pseudoaneurysm in case of chronic atrophic calcific pancreatitis. Pol J Radiol 2016; 81: 462-464.

5. Saad NE, Saad WE, Davies MG, et al. Pseudoaneurysms and the role of minimally invasive techniques in their management. Radiographics 2005; 25 Suppl 1: S173-S189.

6. Kang SS, Labropoulos N, Mansour MA, et al. Expanded indications for ultrasound-guided thrombin injection of pseudoaneurysms. J Vasc Surg 2000; 31: 289-298. 